\title{
Observer-based composite adaptive dynamic terminal sliding-mode controller for nonlinear uncertain SISO systems
}

\author{
Xiaofei Liu, Shengbo Qi*, Reza Malekain, and Zhixiong Li
}

\begin{abstract}
In the present paper, the observer-based composite adaptive terminal sliding-mode control is investigated for the nonlinear uncertain system. First, an adaptive observer is designed to estimate the unavailable high-order derivative of the output. Then, a new dynamic terminal sliding surface is proposed with a state filter, which aims to develop the dynamic terminal sliding mode controller. By the composite adaptive control methods, a new adaptive law is designed, and the stability of the overall system is proofed based on the Lyapunov method. Finally, some numerical simulations are conducted to validate the effectiveness of the proposed algorithm.
\end{abstract}

Keywords: Adaptive control, adaptive observer, dynamic terminal sliding mode control, stability.

\section{INTRODUCTION}

In control systems, sliding mode control (SMC) is a nonlinear control method that alters the dynamics of a nonlinear system to "slide" along a normal crosssection of the system by applying a discontinuous control signals. Many studies on SMC have been conducted. Y. Wei. et al. proposed a output-feedback sliding mode controller for uncertain continuous-time semi-Markovian jump systems which could driving the underlying closedloop system on the sliding surface in finite time [1]. Furthermore, W. Qi et al. designed an observer-based adaptive SMC with applications to DC motor which could effectively reduce the adverse effects of parameter variations and external disturbances [2].

In recent decades, terminal sliding-mode control (TSMC) has been paid increasing attention [3-7]. The traditional sliding mode variable structure control utilizes a linear sliding mode, and the deviation between the system state and a given trajectory converges asymptotically. Compared with the traditional slidingmode control, the TSMC introduces a nonlinear term in the sliding surface function to improve the system's convergence characteristics, so that the system state can converge to a given trajectory within finite time. Therefore, the TSMC has the advantages of fast dynamic response, finite time convergence, and high steady-state tracking accuracy [8]. However, chattering of the control signal and high-frequency noise are main drawbacks of
TSMC due to discontinuous switching term of the control signal and sensitivity to high-frequency noise. To overcome these problems, Liu and Sun [5] proposed a method, namely the dynamic terminal sliding-mode (DTSM) control, in which the time derivative of control input was treated as control variable for the augmented system. The control input becomes chattering-free because of an integrator (like a low-pass filtering) placed in front of the system.

Despite the superiorities of DTSM control, the application of DTSM control is challenging for the following reasons. In non-matching uncertain nonlinear systems, there is no general integral relationship among the system states, and thus the various derivatives of the system state cannot be obtained. Therefore, those sliding mode control methods based on the various orders of the system state can not be achieved [9]. In addition, as like traditional dynamic sliding-mode control $[10,11]$, the augmented system is one dimension larger than the original system in the DTSM control design, and hence the dynamic sliding variable contains an uncertainty term due to the variations of parameters and external disturbance [12]. As a result, tracking errors based on adaptive law will face the same dilemma.

Moreover, unlike the foregoing adaptive law of the observer-based adaptive controller, a provably stable adaptive law employing both the tracking error and the prediction error to update the parameters is proposed,

This article was supported by the National Science Foundation of China (No. 51709248 and 51505475), UOW VC Fellowship, and the National Research Foundation of South Africa (No. IFR160118156967 and RDYR160404161474).

Xiaofei Liu and Shengbo Qi are with College of Engineering, Ocean University of China, Qingdao 266100, China (e-mails: 1xf@stu.ouc.edu.cn \& qsb@ouc.edu.cn). Reza Malekain is with Department of Electrical, Electronic and Computer Engineering, University of Pretoria, Pretoria, 0002, South Africa (reza.malekian@ieee.org). Zhixiong Li is with School of Mechanical, Materials, Mechatronic and Biomedical Engineering, University of Wollongong, Wollongong, NSW 2522, Australia (zhixiong.li@ieee.org).

* Corresponding author. 
which is inspired by the principle of composite adaptive control [13, 14]. In Ref. [12], based on traditional DSM control, a robust observer was proposed to estimate the state-dependent uncertainty in dynamic sliding variable. Another approach called observer-based adaptive control has achieved great development in recent years [15-20]. Compared with some general state estimators proposed in $[21,22]$, the robust observer is employed to estimate the unavailable system states, so the parameters of controller can be adjusted on-line by the adaptive controller in this observer-based adaptive control scheme. In the previous studies, observer-based adaptive controller was used online to adjust control parameters by employing the observation error [16] or the estimation of tracking error solely [19]; however it would chuck away the information of parameters contained in the one not covered. Furthermore, estimation will be slower owing to the very small observer error in general. If high compensation coefficient or high adaption gain is used to deal with this problem, the amplification of the uncertainties contained in the observation errors may lead to chattering.

In this paper, the observer-based adaptive controller is integrated into the dynamic terminal sliding-mode control to overcome the obstacle to the application of DTSM control.

\section{PROBLEM FORMULATION}

We let $R$ denote the real numbers, $R^{n}$ the real nvectors and $R^{n \times m}$ the real $n \times m$ matrices. The norm of matrix $A \in R^{n \times m}$ defined as $\|A\|=\sqrt{\lambda_{\max }\left(A^{T} A\right)}, \lambda_{\max }(A)$ and $\lambda_{\text {min }}(A)$ stand for the maximum and minimum eigenvalues of matrix $A \in R^{n \times m},\|x\|=\sqrt{x_{1}^{2}+\cdots+x_{n}^{2}}$ stands for the norm of vector $x \in R^{n}$.

Consider the $n$ th-order single input single output nonlinear system of the form

$$
\begin{aligned}
& \dot{x}=A x+B[f(x)+g(x) u+d(t)], \\
& y=C x .
\end{aligned}
$$

where $x \in R^{n}$ is the accessible state vector of system, $u \in R^{1}$ is the scalar control input, $y \in R^{1}$ is the scalar output of system, $f(x) \in R^{1}$ and $g(x) \in R^{1}$ are unknown but continuous functions, $d(t) \in R^{1}$ is the external bounded disturbance. The pair $(A, B)$ is controllable, and $B \in R^{n \times 1}$. The row vector $C \in R^{1 \times n}$ is a design parameter chosen such that $(A, C)$ is observable and zeros of system $(A, B, C)$ are all in the open left-half plane.

Because of the system uncertainty (i.e. external disturbance, parameters variation and unmodeled dynamic), the dynamic model of the system is an approximation of the real [23]. Hence in the presence of uncertainties, functions $f(\underline{x})$ and $g(\underline{x})$ can be represented the form as

$$
\begin{aligned}
& f(\underline{x})=\hat{f}(\underline{x})+\Delta f(\underline{x}), \\
& g(\underline{x})=\hat{g}(\underline{x})+\Delta g(\underline{x}) .
\end{aligned}
$$

where $\hat{f}(\underline{x})$ and $\hat{g}(\underline{x})$ are the known parts, and $\Delta f(\underline{x})$ and $\Delta g(\underline{x})$ are the unknown parts of $f(\underline{x})$ and $g(\underline{x})$ respectively. In this paper $\hat{f}(\underline{x}), \hat{g}(\underline{x}), \Delta f(\underline{x}), \Delta g(\underline{x})$ and $d(t)$ satisfy the assumption as follows.

Assumption 1: Assume that $\hat{f}(\underline{x}), \hat{g}(\underline{x}), \Delta f(\underline{x})$, $\Delta g(\underline{x}), d(t)$ and their derivative $\dot{\hat{f}}(\underline{x}), \dot{\hat{g}}(\underline{x}), \Delta \dot{f}(\underline{x})$, $\Delta \dot{g}(\underline{x}), \dot{d}(\underline{x})$ satisfy $|\hat{f}(\underline{x})| \leq F \leq \infty,|\dot{\hat{f}}(\underline{x})| \leq F_{d} \leq \infty$, $0 \leq G_{\min } \leq \hat{g}(\underline{x}) \leq G_{\max }, 0 \leq G_{d \min } \leq \dot{\hat{g}}(\underline{x}) \leq G_{d \max }$ and $|d| \leq d_{H}, \quad|\dot{d}| \leq \dot{d}_{H}$, respectively, for all $\underline{x} \in \Omega_{\underline{x}} \subset R^{n}$, where $F, F_{d}, G_{\min }, G_{\max }, G_{d \text { min }}, G_{d \max }, d_{H}, \dot{d}_{H}$ are positive constants.

Based on (2), system dynamic (1) can be rewritten as follows [15]

$$
\begin{aligned}
& \underline{\dot{x}}=A \underline{x}+B\left[\hat{f}(\underline{x})+\hat{g}(\underline{x}) u+T_{1}\right], \\
& y=C \underline{x} .
\end{aligned}
$$

Here $T_{1}$ is the uncertainty of system dynamic:

$$
T_{1}=\Delta f(\underline{x})+\Delta g(\underline{x}) u+d(t)
$$

From the Assumption 1, and the boundedness of $\mathrm{u}$, we can conclude that $T_{1}$ is bounded.

Suppose that $y_{d}$ is the reference signal, which satisfies the following assumption.

Assumption 2: The signal $y_{d}$ and its derivatives up to $y_{d}^{(n+1)}$ are bounded for all time, and the $(n+1)^{t h}$ derivative $y_{d}^{(n+1)}$ is a continuous function of $t$. Let $e=y-y_{d}$ denote the tracking-error, $E \in R^{n}$ denote $E=\left[\begin{array}{llll}e & \dot{e} & \cdots & e^{(n-1)}\end{array}\right]^{T}$.

In the conventional DTSM control [5], the dynamic terminal sliding variable is defined as

$$
s(t)=K E-K P(t)
$$

where $P(t)=\left[\begin{array}{llll}p(t) & \dot{p}(t) & \cdots & p^{(n-1)}(t)\end{array}\right]^{T} \in R^{n}, p(t)$ is a function designed such that the following assumption holds.

Assumption 3: Consider function $p(t), R_{+} \rightarrow R$, $p(t) \in C^{n}[0, \infty), \dot{p}, \ddot{p}, \cdots, p^{(n)} \in L^{\infty}, \quad p(t)$ is bounded in interval $[0, T]$ for some $T>0$, 
$p(0)=e(0), \quad \dot{p}(0)=\dot{e}(0), \cdots, \quad p^{(n)}(0)=e^{(n)}(0)$.

Moreover, when $t>T, p(t)=0, \dot{p}(t)=0, \cdots$, $p^{(n)}(t)=0 . C^{n}[0, \infty)$ represent the set of all rank differentiable continuous functions defined in $[0, \infty)$. $K=\left[\begin{array}{llll}k_{0} & k_{1} & \cdots & k_{n-1}\end{array}\right] \in R^{1 \times n}$ is a positive vector, defined by the follow:

$$
s(t)=K E-K P(t)=\left(\frac{d}{d t}+\lambda_{0}\right)^{n-1}[e-p(t)]
$$

where $\lambda_{0}$ is a positive design parameter.

Define terminal function $p(t)$ as

$$
p(t)=\left\{\begin{array}{cc}
\sum_{j=0}^{n}\left[\sum_{l=0}^{n}\left(\frac{a_{j l}}{T^{j-l+n+1}} e^{(l)}(0)\right)\right] t^{j+n+1} \\
+\sum_{i}^{n} \frac{1}{i !} e^{(k)}(0) t^{k}, & \text { if } \mathrm{t} \leq \mathrm{T} \\
0, & \text { if } \mathrm{t} \geq \mathrm{T}
\end{array}\right.
$$

The dynamic terminal sliding surface $\delta(t)$ is defined as

$$
\delta(t)=\dot{s}(t)+\lambda_{1} s(t)
$$

where $\lambda_{1}$ is a positive design parameter.

For system (1), the conventional DTSM control used in general design is

$$
\begin{aligned}
\dot{u} & =\omega_{c}=\frac{1}{k_{n} C A^{n-1} B \hat{g}(\underline{x})}\left\{-k_{n-1} C A^{n-1} B\left[\hat{f}(\underline{x})+\hat{g}(\underline{x}) u+\hat{T}_{2}\right]\right. \\
& -k_{n-1} C A^{n} B\left[\hat{f}(\underline{x})+\hat{g}(\underline{x}) u+\hat{T}_{1}\right]-k_{n-1} C A^{n-1} B \underline{x} \\
& -\left(\lambda_{1} k_{n-1}+k_{n-2}\right) y^{(n)}-\left(\lambda_{1} k_{n-2}+k_{n-3}\right) y^{(n-1)}-\cdots \\
& \left.-\left(\lambda_{1} k_{1}+k_{0}\right) \ddot{y}-\lambda_{1} k_{0} \dot{y}+I_{1}+I_{2}+\eta \operatorname{sign}(\delta)\right\}
\end{aligned}
$$

where $\eta$ is a positive design parameter, $I_{1}$ and $I_{2}$ are described as

$$
\begin{aligned}
I_{1}= & k_{n-1} y_{d}^{(n+1)}+\left(\lambda_{1} k_{n-1}+k_{n-2}\right) y_{d}^{(n)}+\cdots \\
& \left(\lambda_{1} k_{1}+k_{0}\right) \ddot{y}_{d}+\lambda_{1} k_{0} \dot{y}_{d} \\
I_{2}= & k_{n-1} p(t)^{(n+1)}+\left(\lambda_{1} k_{n-1}+k_{n-2}\right) p(t)^{(n)}+\cdots \\
& +\left(\lambda_{1} k_{1}+k_{0}\right) \ddot{p}(t)+\lambda_{1} k_{0} \dot{p}(t)
\end{aligned}
$$

Consider a Lyapunov function candidate as follows:

$$
V(t)=\frac{1}{2} \delta^{T} \delta
$$

$$
s(t)=K(E-P)=\sum_{l=0}^{n} k_{l}\left(e^{(l-1)}-p^{(l-1)}\right)
$$

The derivative of $s(t)$ is

$$
\begin{aligned}
\dot{s}(t) & =K(\dot{E}-\dot{P}) \\
& =k_{n}\left(e^{(n)}-p^{(n)}\right)+\sum_{i=1}^{n-1} k_{i}\left(e^{(i)}-p^{(i)}\right)
\end{aligned}
$$

The dynamic terminal sliding surface and its derivative are:

$$
\begin{aligned}
\delta & =k_{n}\left(e^{(n)}-p^{(n)}\right)+\sum_{i=1}^{n-1} k_{i}\left(e^{(i)}-p^{(i)}\right) \\
& +\lambda_{1} \sum_{l=1}^{n} k_{l}\left(e^{(l-1)}-p^{(l-1)}\right) \\
\dot{\delta}= & k_{n}\left(e^{(n+1)}-p^{(n+1)}\right)+\sum_{i=1}^{n-1} k_{i}\left(e^{(i+1)}-p^{(i+1)}\right) \\
& +\lambda_{1} \sum_{l=1}^{n} k_{l}\left(e^{(l)}-p^{(l)}\right)
\end{aligned}
$$

Since

$$
\begin{aligned}
& e^{(n+1)}=\dot{e}^{(n)}=\dot{y}^{(n)}-\dot{y}_{d}^{(n)}=\ddot{y}_{n}-\ddot{y}_{n d}, \\
& k_{n}\left(e^{(n+1)}-p^{(n+1)}\right)=k_{n}\left(\ddot{y}_{n}-\ddot{y}_{n d}-p^{(n+1)}\right) \\
& =k_{n}\left(\dot{f}(x)+\dot{g}(x) u(t)+g(x) \dot{u}(t)+\dot{d}(t)-\ddot{y}_{n d}-p^{(n+1)}\right), \\
& \sum_{i=1}^{n-1} k_{i}\left(e^{(i+1)}-p^{(i+1)}\right) \\
& =k_{n-1}\left(f(x)+g(x) u(t)+d(t)-\dot{y}_{n d}-p^{(n)}\right) \\
& \quad+\sum_{i=1}^{n-2} k_{i}\left(e^{(i+1)}-p^{(i+1)}\right), \\
& \quad \lambda_{1} \sum_{l=1}^{n} k_{l}\left(e^{(l)}-p^{(l)}\right) \\
& =\lambda_{1} k_{n}\left(f(x)+g(x) u(t)+d(t)-\dot{y}_{n d}-p^{(n)}\right) \\
& \quad+\lambda_{1} \sum_{l=1}^{n-1} k_{l}\left(e^{(l)}-p^{(l)}\right) .
\end{aligned}
$$

Therefore

$$
\begin{aligned}
\dot{\delta} & =\left(k_{n} \dot{g}(x)+\left(k_{n-1}+\lambda_{1} k_{n}\right) g(x)\right) u(t) \\
& +\left(k_{n-1}+\lambda_{1} k_{n}\right) d(t)+k_{n}(g(x) \dot{u}(t)+\dot{d}(t)) \\
& +k_{n}\left(\dot{f}(x)-\ddot{y}_{n d}-p^{(n+1)}\right) \\
& +\left(k_{n-1}+\lambda_{1} k_{n}\right)\left(f(x)-\dot{y}_{n d}-p^{(n)}\right) \\
& +\sum_{i=1}^{n-2} k_{i}\left(e^{(i+1)}-p^{(i+1)}\right)+\lambda_{1} \sum_{l=1}^{n-1} k_{l}\left(e^{(l)}-p^{(l)}\right)
\end{aligned}
$$

Substituting the dynamic control law (9) into (17), then the sufficient condition for the existence of DTSM can be obtained as:

$$
\eta>\left\|k_{n-1} C A^{n-1} B T_{2}\right\|+\left\|k_{n-1} C A^{n} B T_{1}\right\|
$$

which ensures the derivative of Lyapunov function $\dot{V}<0$ when $\delta \neq 0$. That means this Lyapunov function 
will decrease gradually [13] and the sliding surface $\delta$ will converge to zero. According to (5) - (8) and Assumption 2, it is easy to know that $s(0)=0, \dot{s}(0)=0$, $\delta(0)=0$. Thus, it implies that $\delta(t) \equiv 0, s(t) \equiv 0$.

Remark 1: It follows from assumptions before that $s(0)=K(E(0)-P(0))=0$,

$\dot{s}(0)=K(\dot{E}(0)-\dot{P}(0))=0$.

From (8), we can get $\delta(0)=0$. According to Lyapunov analysis, $\delta(0)=0$ can be achieved. Consider $\delta(0)=0$ and (8), we have $s(0)=0$ for all the time. Then, if we choose $P(t)(\forall t \geq T)$, the tracking error $E(t)(\forall t \geq T)$ will converge to zero in finite time $T$.

From (9) - (11), it can be seen that the control law contains the high-order derivative of the output $y^{(n)}$. And system described in this paper as show as follows:

$$
\begin{aligned}
& y=C \underline{x}, \\
& \dot{y}=C A \underline{x}, \\
& \vdots \\
& y^{(n-1)}=C A^{n-1} \underline{x}, \\
& y^{(n)}=C A^{n} \underline{x}+C A^{n-1} B\left[\hat{f}(\underline{x})+\hat{g}(\underline{x}) u+T_{1}\right] .
\end{aligned}
$$

The high-order derivative of the output $y^{(n)}$ cannot be available, since it contains the uncertain component $T_{1}$. However, system state $\underline{x}$ can be measured exactly and the system input $u$ can be obtained directly by system controller. Furthermore, from Assumption 3 and (5), it can be known that the design for function $p(t)$ needs the initial value of $e^{(n)}$. Since $y^{(n)}$ cannot be obtained directly, it can't get the exact value of $e^{(n)}(0)$. As a result, the original DTSM control cannot guarantee that the initial value of dynamic terminal sliding surface satisfies $\delta(0)=0$.

From the discussions above, it is clear that the original dynamic terminal sliding mode control is not applicable to the system described in this paper. To overcome these problems, a new observer-based adaptive dynamic terminal sliding mode (ADTSM) control is designed in the following content.

\section{ADAPTIVE OBSERVER DESIGN}

In this section an adaptive observer based on algebraic Lyapunov equation $[18,24]$ is proposed to estimate the unavailable high-order derivative of the output $y^{(n)}$.

Define a new two-dimensional state as

$$
q=\left[\begin{array}{c}
y^{(n-1)} \\
y^{(n)}
\end{array}\right] \in R^{2}
$$

where, the first component is accessible for evaluation, but the second one is not accessible due to the uncertainties in (19). From (20), estimated value of $y^{(n)}$ can be obtained by estimating the state $q$.

Taking the time derivative of (20), can get the dynamic of state $q$

$$
\begin{aligned}
& \dot{q}=A_{2} q+B_{2}\left\{C A^{n+1} x+C A^{n} B\left[\hat{f}(x)+\hat{g}(x) u+T_{1}\right]\right. \\
& \left.+C A^{n-1} B\left[\dot{\hat{f}}(x)+\dot{\hat{g}}(x) u+\hat{g}(x) \omega+T_{2}\right]\right\}, \\
& y^{(n-1)}=C_{2} q .
\end{aligned}
$$

where $\omega=\dot{u}$ is the dynamic of system input, and system matrices described as

$$
A_{2}=\left[\begin{array}{ll}
0 & 1 \\
0 & 0
\end{array}\right], \quad B_{2}=\left[\begin{array}{l}
0 \\
1
\end{array}\right], \quad C_{2}^{T}=\left[\begin{array}{l}
1 \\
0
\end{array}\right]
$$

$T_{1}$ and $T_{2}$ are unknown uncertainties defined as follows [20]

$$
\begin{aligned}
& T_{1}=\Delta f(\underline{x})+\Delta g(\underline{x}) u+d, \\
& T_{2}=\dot{T}_{1}=\Delta \dot{f}(x)+\Delta \dot{g}(x) u+\Delta g(x) \omega+\dot{d} .
\end{aligned}
$$

To estimate the state $q$, the following adaptive observer is proposed:

$$
\begin{aligned}
& \dot{\hat{q}}=A_{2} \hat{q}+B_{2}\left\{C A^{n+1} x+C A^{n} B\left[\hat{f}(x)+\hat{g}(x) u+\hat{T}_{1}\right]\right. \\
& \left.\quad+C A^{n-1} B\left[\dot{\hat{f}}(x)+\dot{\hat{g}}(x) u+\hat{g}(x) \omega+\hat{T}_{2}\right]\right\} \\
& \quad+\lambda_{2} \Delta_{\lambda_{2}}^{-1} Q^{-1} C^{T} C \tilde{q}, \\
& \hat{y}^{(n-1)}=C_{2} \hat{q} .
\end{aligned}
$$

where $\Delta_{\lambda_{2}}=\operatorname{diag}\left[1, \frac{1}{\lambda_{2}}\right]$ is a positive constant.

$\hat{q}, \hat{T}_{1}, \hat{T}_{2}$ are estimated value of $q, T_{1}, T_{2}$ respectively and updated on-line by adaptive law designed in section 5. $\tilde{q}$ is the observation error vector defined as $\tilde{q}=q-\hat{q}=\left[\begin{array}{ll}\tilde{y}^{(n-1)} & \tilde{y}^{(n)}\end{array}\right]^{T} . \quad Q \quad$ is the unique solution of the following Lyapunov equation:

$$
Q+A_{2}^{T} Q+Q A_{2}-C_{2}^{T} C_{2}=0
$$

The explicit solution of (16)can be obtained as

$$
Q(i, j)=(-1)^{i+j} l_{i+j-2}^{-1}, 1 \leq i, j \leq 2
$$


where $l_{n}^{r}=\frac{n !}{(n-r) ! r !}$

Furthermore, $Q$ is a symmetric positive definite matrix [24].

Theorem 1: Supposed that the system satisfies Assumptions (1) and (2), and $\hat{T}_{1}, \hat{T}_{2}$ are bounded. The observer given by (23)-(25) can ensure that the observation error $\tilde{q}$ is uniformly bounded and converges to $\Omega_{\tilde{q}}$ defined as

$$
\Omega_{\tilde{q}}=\left\{\tilde{q}\|\tilde{q}\| \leq \frac{2\left\|B_{2}^{T} Q\right\|\left(C A^{n} B\left|\tilde{T}_{2}\right|\right)}{\lambda_{\min }(Q)+\lambda_{2}}\right\}
$$

Moreover, the radius of set $\Omega_{\tilde{q}}$ can be made arbitrarily small, if $\lambda_{2}$ is chosen to be sufficiently large.

Proof: From (21) and (23), the observation error dynamic can be written as

$$
\dot{\tilde{q}}=A_{2} \tilde{q}+B_{2}\left(C A^{n} B \tilde{T}_{1}+C A^{n-1} B \tilde{T}_{2}\right)-\lambda_{2} \Delta_{\lambda_{2}}^{-1} Q^{-1} C_{2}^{T} C_{2} \tilde{q}
$$

here $\tilde{T}_{1}=T_{1}-\hat{T}_{1}, \quad \tilde{T}_{2}=T_{2}-\hat{T}_{2}$. Since $T_{1}, T_{2}, \hat{T}_{1}$, $\hat{T}_{2}$ are bounded and the closed-loop system (27) is stable, $\tilde{T}_{1}, \tilde{T}_{2}$ and $\tilde{q}$ are also bounded.

Consider the Lyapunov function as

$$
V_{0}=\frac{1}{2} \tilde{q}^{T} Q \tilde{q}
$$

The derivation of (28) along (27) is given by

$$
\begin{aligned}
\dot{V}_{0}= & \frac{1}{2} \dot{\tilde{q}}^{T} Q \tilde{q}+\frac{1}{2} \tilde{q}^{T} Q \dot{\tilde{q}} \\
= & -\frac{1}{2} \tilde{q}^{T} Q A_{2}^{T} \tilde{q}+B_{2}^{T} Q \tilde{q}\left(C A^{n} B \tilde{T}_{1}+C A^{n-1} B \tilde{T}_{2}\right) \\
& -\frac{1}{2} \lambda_{2} \tilde{q}^{T} Q \Delta_{\lambda_{2}}^{-1} Q^{-1} C_{2}^{T} C_{2} \tilde{q}-\frac{1}{2} \lambda_{2} \tilde{q}^{T} C_{2}^{T} C_{2} Q^{-1} \Delta_{\lambda_{2}}^{-1} Q \tilde{q} \\
& +\frac{1}{2} \lambda_{2} \tilde{q}^{T} C_{2}^{T} C_{2} \tilde{q}
\end{aligned}
$$

Because of

$$
\begin{aligned}
\tilde{q}^{T} Q \Delta_{\lambda_{2}}^{-1} Q^{-1} C_{2}^{T} C_{2} \tilde{q} & =\tilde{q}^{T} C_{2}^{T} C_{2} Q^{-1} \Delta_{\lambda_{2}}^{-1} Q \tilde{q} \\
& =\tilde{q}^{T} C_{2}^{T} C_{2} \tilde{q}=\left(y^{(n-1)}\right)^{2}
\end{aligned}
$$

(29) can be re-arranged as:

$$
\begin{aligned}
\dot{V}_{0} & =-\frac{1}{2} \tilde{q}^{T} Q A_{2}^{T} \tilde{q}+B_{2}^{T} Q \tilde{q}\left(C A^{n} B \tilde{T}_{1}+C A^{n-1} B \tilde{T}_{2}\right) \\
& -\frac{1}{2} \lambda_{2}\left(y^{(n-1)}\right)^{2}
\end{aligned}
$$

$$
\begin{aligned}
& \text { Since }\left|y^{(n-1)}\right| \leq\|\tilde{q}\|, \\
& \dot{V}_{0} \leq-\frac{1}{2}\|\tilde{q}\|\left\{\left[\lambda_{\min }(Q)+\lambda_{2}\right]\|\tilde{q}\|-2\left\|B_{2}^{T} Q\right\|\left(C A^{n} B\left|\tilde{T}_{1}\right|+C A^{n+1} B\left|\tilde{T}_{2}\right|\right)\right\}
\end{aligned}
$$

Obviously, $\dot{V}_{0}$ is negative as long as $\tilde{q}$ is outside of the compact set $\Omega_{\tilde{q}}$ defined as

$$
\Omega_{\tilde{q}}=\left\{\tilde{q}\|\tilde{q}\| \leq \frac{2\left\|B_{2}^{T} Q\right\|\left(C A^{n} B\left|\tilde{T}_{1}\right|+C A^{n-1} B\left|\tilde{T}_{2}\right|\right)}{\lambda_{\min }(Q)+\lambda_{2}}\right\}
$$

According to Lyapunov theorem [13], it can be concluded that $\tilde{q}$ is bounded and converges to $\Omega_{\tilde{q}}$. Moreover, the radius of the set $\Omega_{\tilde{q}}$ can be made arbitrarily small, if $\lambda_{2}$ is chosen to be sufficiently large.

\section{ADTSM CONROLLER DESIGN}

According to the discussion in section $2, \hat{p}(t)$ cannot be designed and the initial state of $\delta(t)$ cannot be made satisfy $\delta(0)=0$, due to the unavailable state $y^{(n)}$. To deal with this problem, a new terminal sliding-mode surface is designed:

$$
\delta_{f}(t)=L(p)\left[\dot{\hat{s}}(t)+\lambda_{3} \hat{s}(t)\right] \in R^{1}
$$

where $L(p)=\lambda_{f} /\left(p+\lambda_{f}\right), L(p)$ is a state filter, $p$ is the Laplace operator, $\lambda_{3}$ and $\lambda_{f}$ are positive parameters. $\hat{s}$ is a new terminal sliding mode, defined as:

$$
\hat{s}=K E-K \hat{P}(t) \in R^{1}
$$

In (34), $K$ and $E$ are the same with (5) and (6), $\hat{p}(t)$ is a design function which satisfies the following assumption.

Assumption 4: Consider the function $\hat{p}(t): R_{+} \rightarrow R$, $\hat{p}(t) \in C^{n}[0, \infty), \dot{\hat{p}}, \ddot{\hat{p}}, \cdots, \hat{p}^{(n)} \in L^{\infty} . \hat{p}(t)$ is a bounded in interval $[0, T]$ for some $T>0, \hat{p}(0)=e(0)$, $\dot{\hat{p}}(0)=\dot{e}(0), \cdots, \hat{p}^{(n-1)}(0)=e^{(n-1)}(0)$, moreover, when $\quad t>T, \quad \hat{p}(t)=0 \quad, \quad \dot{\hat{p}}(t)=0 \quad, \quad \cdots$, $\hat{p}^{(n-1)}(t)=0 . C^{n}[0, \infty)$ represents the set of all rank continuously differentiable functions defined in $[0, \infty)$.

In this paper, the function $\hat{p}(t)$ chosen as follows

$$
\hat{p}(t)\left\{\begin{array}{c}
\sum_{i=0}^{n-1} \frac{1}{i !} e^{(i)}(0) t^{i}+\sum_{j=0}^{n-1}\left[\sum_{l=0}^{n-1} \frac{a_{j l}}{T^{j-l+n+1}} e^{(l)}(0)\right] t^{j+n+1}, \text { if } 0 \leq t \leq T \\
0 \quad, \text { if } t>T
\end{array}\right.
$$


where $a_{j l}$ can be obtained by Assumption 4 .

Since $L(p)=\lambda_{f} /\left(p+\lambda_{f}\right)$,(33) can be rewritten as

$$
\delta_{f}(t)=\lambda_{f} \hat{s}(t)+\frac{\lambda_{f} \lambda_{2}-\lambda_{f}^{2}}{p+\lambda_{f}} \hat{s}(t)
$$

From Assumption 4 and (34), it can be known that $y^{(n)}$ and its initial value $y^{(n)}(0)$ do not exist in $\hat{p}(t)$ and $\hat{s}(t)$. Since $y^{(i)}(i=1,2, \cdots, n-1)$ and their initial values $y^{(i)}(0)(i=1,2, \cdots, n-1)$ are available, $\hat{p}(t)$ and $\hat{s}(t)$ can be exactly calculated by (35) and (34), thus $\delta_{f}(t)$ is available. Moreover, $\delta_{f}(0)=0$, if Assumption 4 holds. Consider the control law:

$$
\dot{u}_{A}=\omega_{A}=\omega_{A c}+\omega_{A r}
$$

The term $\omega_{A c}$ is the adaptive control, which is designed based on the observed state $\hat{y}^{(n)}$ and the adjustable parameters $\hat{T}_{1}$ and $\hat{T}_{2}$.

$$
\begin{aligned}
\omega_{A c} & =\frac{1}{k_{n} C A^{n-1} B \hat{g}(x)}\left\{-k_{n-1} C A^{n-1} B\left[\dot{\hat{f}}(x)+\dot{\hat{g}}(x) u+\hat{T}_{2}\right]\right. \\
& -k_{n-1} C A^{n} B\left[\hat{f}(x)+\hat{g}(x) u+\hat{T}_{1}\right]-k_{n-1} C A^{n+1} B x \\
& -\left(\lambda_{3} k_{n-1}+k_{n-2}\right) \hat{y}^{(n)}-\left(\lambda_{3} k_{n-2}+k_{n-3}\right) y^{(n-1)} \\
& \left.-\cdots-\left(\lambda_{3} k_{1}+k_{0}\right) \ddot{y}-\lambda_{3} k_{0} \dot{y}+I_{A 1}+I_{A 2}\right\}
\end{aligned}
$$$$
I_{1}=k_{n-1} y_{d}^{(n-1)}+\left(\lambda_{3} k_{n-1}+k_{n-2}\right) y_{d}^{(n)}+\cdots+\left(\lambda_{3} k_{1}+k_{0}\right) \ddot{y}_{d}
$$$$
+\lambda_{3} k_{0} \dot{y}_{d}
$$$$
I_{A 2}=k_{n-1} \hat{p}^{(n+1)}(t)+\left(\lambda_{3} k_{n-1}+k_{n-2}\right) \hat{p}^{(n)}(t)+
$$

$$
\cdots+\left(\lambda_{3} k_{1}+k_{0}\right) \ddot{\hat{p}}(t)+\lambda_{3} k_{0} \dot{\hat{p}}(t)
$$

where $\hat{T}_{1}$ and $\hat{T}_{2}$ are estimated values of $T_{1}$ and $T_{2}$ respectively. A composite adaptive law will be derived in section 5.

The compensated control $\omega_{A r}$ is employed to guarantee the dynamic terminal-sliding surface to be an invariant set by compensating the system uncertainties and the external disturbance. In this paper, $\omega_{A r}$ is chosen such as:

$$
\omega_{A r}=-\frac{1}{k_{n} C A^{n-1} B \hat{g}(x)} \beta \delta_{f}
$$

where $\beta$ is a positive design parameter.

\section{COMPOSITE ADAPTIVE LAW DESIGN}

In this section, a new adaptive law which extracts parameters information from both observation errors and tracking errors is developed. In the following, the observation errors and tracking errors are presented based on adaptive law, and the composite adaptive law is generalized.

\subsection{Observation errors based adaptive law design}

Observation errors based on adaptive law is the most common employed adaptive law in observer-based adaptive control [25] which based on the observation error vector $\tilde{q}$. However, in system (1), the state of $\tilde{q}$ is unavailable, so the $\tilde{q}$ cannot be used to design adaptive law directly. To deal with this problem, inspired by the works in Ref. [26], a new state $\tilde{q}_{f}$ is introducted as follows

$$
\tilde{q}_{f}=L(p) \tilde{q}
$$

where $\quad \tilde{q}_{f}=\left[\begin{array}{ll}\tilde{y}_{f}^{(n-1)} & \tilde{y}_{f}^{(n)}\end{array}\right]^{T}, L(p)=\lambda_{f} /\left(p+\lambda_{f}\right)$.

$L(p)$ is a state filter as same as the filter in (33). From (27) and (42), (42) can be expressed as

$$
\begin{aligned}
\dot{\tilde{q}}_{f} & =A_{2} \tilde{q}+B_{2}\left(C A^{n} B \tilde{T}_{1}+C A^{n-1} B \tilde{T}_{2}+w_{1}\right) \\
& -\lambda_{2} \Delta_{\lambda_{2}}^{-1} Q^{-1} C^{T} C \tilde{q}_{f}
\end{aligned}
$$

where

$w_{1}=-\left(C A^{n} B \tilde{T}_{1}+C A^{n-1} B \tilde{T}_{2}\right)+L(p)\left(C A^{n} B \tilde{T}_{1}+C A^{n-1} B \tilde{T}_{2}\right)$

Since $L(p)=\lambda_{f} /\left(p+\lambda_{f}\right)$, the $\tilde{y}_{f}^{(n-1)}$ and $\tilde{y}_{f}^{(n)}$ can be expressed as

$$
\begin{aligned}
& \tilde{y}_{f}^{(n-1)}=\left[\lambda_{f} /\left(p+\lambda_{f}\right)\right] \tilde{y}^{(n-1)}, \\
& \tilde{y}_{f}^{(n)}=\lambda_{f} \tilde{y}^{(n-1)}-\left[\lambda_{f}^{2} /\left(p+\lambda_{f}\right)\right] \tilde{y}^{(n-1)} .
\end{aligned}
$$

The system state $y^{(n-1)}$ is available and $\hat{y}^{(n-1)}$ can be obtained directly, so $\tilde{y}_{f}^{(n)}$ is available, then $\tilde{y}_{f}^{(n-1)}, \quad \tilde{y}_{f}^{(n)}$ and $\tilde{q}_{f}$ are available. Therefore, the observation errors based on adaptive law can be designed as

$$
\begin{aligned}
& \dot{\hat{T}}_{1}=\gamma_{1} B_{2}^{T} Q M \tilde{q}_{f} C A^{n} B, \\
& \dot{\hat{T}}_{2}=\gamma_{2} B_{2}^{T} Q M \tilde{q}_{f} C A^{n-1} B .
\end{aligned}
$$

where $\gamma_{i}>0,(i=1,2)$ is adaptive gain. In this paper, $\gamma_{i}$ is chosen as appropriate positive constant. $M=\theta I,(\theta \geq 1)$ is a compensation gain matrix, which used to compensate for the filter-induced signal attenuation. Here $\theta$ is a positive design parameter, which is satisfied $\theta \geq 1$. $I$ is the identity matrix.

\subsection{Tracking error based adaptive law design}

From (33) and (34), it is clear that the new terminal sliding mode surface $\delta_{f}(t)$ contains the tracking errors. 
In this section, $\delta_{f}(t)$ is used to design the adaptive law.

Taking the time derivative of $\delta_{f}$ gives the dynamic of $\delta_{f}$ as follows:

$$
\begin{aligned}
\dot{\delta}_{f}= & L(p)\left\{k_{n-1} C A^{n-1} B\left[\dot{\hat{f}}(x)+\dot{\hat{g}}(x) u+\hat{T}_{2}\right]\right. \\
& +k_{n-1} C A^{n} B\left[\hat{f}(x)+\hat{g}(x) u+\hat{T}_{1}\right]+k_{n-1} C A^{n+1} B x \\
& +\left(\lambda_{3} k_{n-1}+k_{n-2}\right) y^{(n)}+\left(\lambda_{3} k_{n-2}+k_{n-3}\right) y^{(n-1)} \\
& \left.+\cdots+\left(\lambda_{3} k_{1}+k_{0}\right) \ddot{y}+\lambda_{3} k_{0} \dot{y}-I_{A 1}-I_{A 2}\right\}
\end{aligned}
$$

where, $I_{A 1}$ and $I_{A 2}$ have the same definition as (39) and (40). Taking the adaptive control law (38) - (41) into (47), we have:

$$
\begin{aligned}
\dot{\delta}_{f} & =k_{n-1} C A^{n} B \hat{T}_{1}+k_{n-1} C A^{n-1} B \hat{T}_{2}+\left(\lambda_{3} k_{n-1}+k_{n-2}\right) \tilde{y}_{f}^{(n)} \\
& -\beta \delta_{f}+w_{2}
\end{aligned}
$$

where

$$
\begin{aligned}
w_{2}= & -\left[k_{n-1} C A^{n} B \tilde{T}_{1}+k_{n-1} C A^{n-1} B \tilde{T}_{2}+k_{n-1} C A^{n-1} B \hat{g}(x) \omega_{r}\right] \\
& +L(p)\left[k_{n-1} C A^{n} B \tilde{T}_{1}+k_{n-1} C A^{n-1} B \tilde{T}_{2}\right. \\
& \left.+k_{n-1} C A^{n-1} B \hat{g}(x) \omega_{r}\right]
\end{aligned}
$$

Since $\delta_{f}$ is available, the tracking-error-based adaptive law of parameters $\hat{T}_{1}, \hat{T}_{2}$ can be designed as follows

$$
\begin{aligned}
& \dot{\hat{T}}_{1}=\gamma_{3} k_{n-1} \delta_{f} C A^{n} B, \\
& \dot{\hat{T}}_{2}=\gamma_{4} k_{n-1} \delta_{f} C A^{n-1} B .
\end{aligned}
$$

where $\gamma_{j}>0,(j=3,4)$ is adaption gain, in this paper,

$\gamma_{j}$ is chosen as appropriate positive constants.

\subsection{Composite adaptive law design}

In this section, inspired by the composite adaptive control methods [27, 28], a fast, stable and relatively smooth adaptive law which extracts parameter information from both the tracking-error and the observed-error is proposed. The new adaptive law is designed as

$$
\begin{aligned}
& \dot{\hat{T}_{1}}=\gamma_{c 1}\left(B_{2}^{T} \Delta_{\gamma} Q M_{c} \tilde{q}_{f} C A^{n} B+k_{n-1} \delta_{f} C A^{n} B\right)-\sigma_{1} \hat{T}_{1} \\
& \dot{\hat{T}_{2}}=\gamma_{c 2}\left(B_{2}^{T} \Delta_{\lambda} Q M_{c} \tilde{q}_{f} C A^{n-1} B+k_{n-1} \delta_{f} C A^{n-1} B\right)-\sigma_{2} \hat{T}_{2}
\end{aligned}
$$

where $\gamma_{c 1}, \gamma_{c 2}$ are adaption gains chosen as appropriate positive constants. $M_{c}=\theta_{c} I\left(\theta_{c} \geq 1\right)$ is a compensation gain matrix, where $\theta_{c}$ is the design parameter satisfied $\theta_{c} \geq 1$. I is an identity matrix. $\sigma_{1}, \sigma_{2}$ are positive design parameters as the $\sigma-$ modification employed to avoid the parameter shift problem $[29,30]$ defined in next section.

\section{STABILITY ANALYSIS}

In this section, the stability of proposed adaptive controller is analyzed. The following lemma is required in the stability analysis.

Lemma 1: If Assumptions 1 and 2 are satisfied, then there exists positive constants $c_{1}, c_{2}$ and $c_{3}$ such as:

(a) $\left\|w_{1}\right\| \leq c_{1}\left|\tilde{T}_{1}\right|+c_{2}\left|\tilde{T}_{2}\right|$

(b) $\left\|w_{2}\right\| \leq c_{1}\left|\tilde{T}_{1}\right|+c_{2}\left|\tilde{T}_{2}\right|+c_{3}$

Proof: Equations (44) and (49) can be arranged as follows

$$
\begin{aligned}
w_{1}= & {\left[-k_{n-1} C A^{n} B \tilde{T}_{1}+L(p)\left(k_{n-1} C A^{n} B \tilde{T}_{1}\right)\right]+} \\
& {\left[-k_{n-1} C A^{n-1} B \tilde{T}_{2}+L(p)\left(k_{n-1} C A^{n-1} B \tilde{T}_{2}\right)\right] } \\
w_{2}= & {\left[-k_{n-1} C A^{n} B \tilde{T}_{1}+\left(L(p) k_{n-1} C A^{n} B \tilde{T}_{1}\right)\right] } \\
& +\left[-k_{n-1} C A^{n-1} B \tilde{T}_{2}+L(p)\left(k_{n-1} C A^{n-1} B \tilde{T}_{2}\right)\right] \\
& +\left\{-k_{n-1} C A^{n-1} B \hat{g}(x) \omega_{r}+L(p)\left[k_{n-1} C A^{n-1} B \hat{g}(x) \omega_{r}\right]\right\}
\end{aligned}
$$

From (56) and (57),

$$
\begin{aligned}
\left|w_{1}\right| \leq & \left\|-k_{n-1} C A^{n} B \tilde{T}_{1}+L(p)\left(k_{n-1} C A^{n} B \tilde{T}_{1}\right)\right\|+\|-k_{n-1} C A^{n-1} B \tilde{T}_{2} \\
& +L(p)\left(k_{n-1} C A^{n-1} B \tilde{T}_{2}\right) \|
\end{aligned}
$$

$$
\begin{aligned}
\left|w_{2}\right| \leq & \left\|-k_{n-1} C A^{n} B \tilde{T}_{1}+L(p)\left(k_{n-1} C A^{n} B \tilde{T}_{1}\right)\right\|+\|-k_{n-1} C A^{n-1} B \tilde{T}_{2} \\
& +L(p)\left(k_{n-1} C A^{n-1} B \tilde{T}_{2}\right)\|+\|-k_{n-1} C A^{n-1} B \hat{g}(x) \omega_{r} \\
& +L(p)\left[k_{n-1} C A^{n-1} B \hat{g}(x) \omega_{r}\right] \|
\end{aligned}
$$

Since the system (1) satisfied Assumptions 1 and 2, $L(p)$ is a stable filter, and $\omega_{r}$ is bounded. It is clear that there exist positive constants $c_{1}, c_{2}$ and $c_{3}$ such as:

$$
\begin{aligned}
& \left\|-k_{n-1} C A^{n} B \tilde{T}_{1}+L(p)\left(k_{n-1} C A^{n} B \tilde{T}_{1}\right)\right\| \leq c_{1}\left|\tilde{T}_{1}\right|, \\
& \left\|-k_{n-1} C A^{n-1} B \tilde{T}_{2}+L(p)\left(k_{n-1} C A^{n-1} B \tilde{T}_{2}\right)\right\| \leq c_{2}\left|\tilde{T}_{2}\right|, \\
& \left\|-k_{n-1} C A^{n-1} B \hat{g}(x) \omega_{r}+L(p)\left[k_{n-1} C A^{n-1} B \hat{g}(x) \omega_{r}\right]\right\| \leq c_{3} .
\end{aligned}
$$

Using the inequalities above, (58) and (59) can be bounded by 


$$
\begin{aligned}
& \left\|w_{1}\right\| \leq c_{1}\left|\tilde{T}_{1}\right|+c_{2}\left|\tilde{T}_{2}\right|, \\
& \left\|w_{2}\right\| \leq c_{1}\left|\tilde{T}_{1}\right|+c_{2}\left|\tilde{T}_{2}\right|+c_{3} .
\end{aligned}
$$

Lemma 2: If the sliding mode surface is defined as $s(t)=\left(d / d t+\lambda_{s}\right)^{n-1} e$, where $e=x-x_{d}$ is the tracking error, and $n-1$ is the relative degree of the system. Bounds of $s$ can be directly translated into bounds of tracking error vector $E=\left[e \dot{e} \cdots e^{(n-1)}\right]^{T}$. Specifically, if

$$
\begin{aligned}
& E(0)=0, \text { then } \\
& \forall t \geq 0,|s(t)| \leq \phi \Rightarrow \\
& \forall t \geq 0,\left|e^{(t)}(t)\right| \leq \phi / \lambda_{s}^{n-1-i},(i=1,2 \cdots n-1)
\end{aligned}
$$

here $\phi \geq 0$ is the up-bound of the sliding surface $|s(t)|$. Finally, the theorem 2 can be established.

Theorem 2: Consider the nonlinear system (1) with the control law given by (38)-(41) and the observer given by (23) which estimating the unavailable state $y^{(n)}$. Let the parameters $\hat{T}_{1}$ and $\hat{T}_{2}$ be adjusted by the composite adaptive laws (52) and (53). If Assumptions 1 and 2 are satisfied, the design parameters are selected such as: $\lambda_{2}>\bar{\lambda}_{2} / \theta_{c}, \sigma_{1}>2 \bar{\sigma}_{1}, \sigma_{2}>2 \bar{\sigma}_{2}, \beta>\bar{\beta}$, where $\bar{\lambda}_{2}, \theta_{c}, \bar{\sigma}_{1}, \bar{\sigma}_{2}$ and $\bar{\beta}$ are constants defined later. Then $\tilde{T}_{1}, \tilde{T}_{2}, \tilde{q}, u, w$ and $e^{(t)}(t),(i=1,2, \cdots, n-1)$ are uniformly ultimately bounded (UUB), moreover, the tracking error converges to following bound in finite time $\mathrm{T}$.

$$
\left|e^{(t)}(t) \leq \rho\left(\frac{-\alpha_{8}(t)+\sqrt{\alpha_{8}^{2}(t)+4 \beta \alpha_{9}(t)}}{2 \beta \lambda_{1} \lambda_{0}^{i}}\right)\right|
$$

here $(i=1,2, \cdots n-1), \forall t \geq T$.

Proof: Consider the Lyapunov function candidate:

$$
V=V_{1}+V_{2}+V_{3}
$$

(62)

Where

$$
\begin{aligned}
& V_{1}=\frac{1}{2} \tilde{q}_{f}^{T} M_{C} Q \tilde{q}_{f} \\
& V_{2}=\frac{1}{2} \delta_{f}^{2} \\
& V_{3}=\frac{1}{2 \gamma_{c 1}} \tilde{T}_{1}^{2}+\frac{1}{2 \gamma_{c 2}} \tilde{T}_{2}^{2}
\end{aligned}
$$

(65)

The derivation of $V_{1}$ along system (43) and (24) is given by

$$
\begin{aligned}
\dot{V}_{1}= & \frac{1}{2} \dot{\tilde{q}}_{f}^{T} M_{C} Q \tilde{q}_{f}+\frac{1}{2} \tilde{q}_{f}^{T} M_{C} Q \dot{\tilde{q}}_{f} \\
= & -\frac{1}{2} \tilde{q}_{f}^{T} M_{C} Q \tilde{q}_{f}+B_{2}^{T} M_{C} Q \tilde{q}_{f}\left(C A^{n} B \tilde{T}_{1}+C A^{n-1} B \tilde{T}_{2}+w_{1}\right) \\
& -\frac{1}{2} \lambda_{2} \tilde{q}_{f}^{T} M_{C} Q \Delta_{\lambda_{2}}^{-1} Q^{-1} C_{2}^{T} C_{2} \tilde{q}_{f}-\frac{1}{2} \lambda_{2} \tilde{q}_{f}^{T} C_{2}^{T} C_{2} Q^{-1} \Delta_{\lambda_{2}}^{-1} M_{C} Q \tilde{q}_{f} \\
& +\frac{1}{2} \lambda_{2} \tilde{q}_{f}^{T} M_{C} C_{2}^{T} C_{2} \tilde{q}_{f}
\end{aligned}
$$

$$
\begin{aligned}
& \text { Since } \\
& \tilde{q}_{f}^{T} M_{C} Q \Delta_{\lambda_{2}}^{-1} Q^{-1} C_{2}^{T} C_{2} \tilde{q}_{f}=\tilde{q}_{f}^{T} C_{2}^{T} C_{2} Q^{-1} \Delta_{\lambda_{2}}^{-1} M_{C} Q \tilde{q}_{f} \\
& =\tilde{q}_{f}^{T} M_{C} C_{2}^{T} C_{2} \tilde{q}_{f}=\theta_{c}\left(y_{f}^{(n-1)}\right)^{2}
\end{aligned}
$$

(66) can be rearranged as

$$
\begin{aligned}
\dot{V}_{1} & =-\frac{1}{2} \tilde{q}_{f}^{T} M_{C} Q A_{2}^{T} \tilde{q}_{f}+B_{2}^{T} M_{C} Q \widehat{q}_{f}\left(C A^{n} B \tilde{T}_{1}+C A^{n-1} B \tilde{T}_{2}+w_{1}\right) \\
& -\frac{1}{2} \lambda_{2} \theta_{c}\left(y_{f}^{(n-1)}\right)^{2}
\end{aligned}
$$

Based on (48), the derivation of $V_{2}$ can be written as

$$
\begin{aligned}
\dot{V}_{2} & =\delta_{f} \dot{\delta}_{f} \\
& =\delta_{f}\left[k_{n-1} C A^{n} B \tilde{T}_{1}+k_{n-1} C A^{(n-1)} B \tilde{T}_{2}\right. \\
& \left.+\left(\lambda_{3} k_{n-1}+k_{n-2}\right) \tilde{y}_{f}^{(n)}+w_{2}-\beta \delta_{f}\right]
\end{aligned}
$$

Because of $\quad \dot{\tilde{T}}_{1}=\dot{T}_{1}-\dot{\hat{T}}_{1}, \quad \dot{\tilde{T}}_{2}=\dot{T}_{2}-\dot{\hat{T}}_{2} \quad$ the differentiation of $V_{3}$ is written as follows

$$
\dot{V}_{3}=-\frac{1}{\gamma_{c 1}} \dot{\hat{T}} \tilde{T}_{1}-\frac{1}{\gamma_{c 2}} \dot{\hat{T}} \tilde{T}_{2}+\frac{1}{\gamma_{c 1}} \dot{T_{1}} \tilde{T}_{1}+\frac{1}{\gamma_{c 2}} \dot{T_{2}} \tilde{T}_{2}
$$

When $T_{1}, T_{2}$ are arbitrarily large and slowly varying with time, the last two terms can be negligible [23].

Based on (62) - (69), and by substituting the composite adaptive laws (52) and (53), the time derivative of $V$ can be described as

$$
\begin{aligned}
\dot{V} & =\dot{V}_{1}+\dot{V}_{2}+\dot{V}_{3} \\
= & -\frac{1}{2} \tilde{q}_{f}^{T} M_{C} Q \tilde{q}_{f}+B_{2}^{T} M_{C} Q \tilde{q}_{f} w_{1}-\frac{1}{2} \theta_{c} \lambda_{2}\left(y_{f}^{(n-1)}\right)^{2} \\
& +\delta_{f}\left(\lambda_{3} k_{n-1}+k_{n-2}\right) \tilde{y}_{f}^{(n)}+\delta_{f} w_{2}-\beta \delta_{f}^{2}+\sigma_{1} \hat{T}_{1} \tilde{T}_{1} \\
& +\sigma_{2} \hat{T}_{2} \tilde{T}_{2}+\frac{1}{\gamma_{c 1}} \dot{T}_{1} \tilde{T}_{1}+\frac{1}{\gamma_{c 2}} \dot{T}_{2} \tilde{T}_{2}
\end{aligned}
$$

Since $\left\|B_{2}^{T} M_{C} Q\right\|=\theta_{c}\left\|B_{2}^{T} Q\right\| \quad, \quad\left|y_{f}^{(n-1)}\right| \leq\left\|\tilde{q}_{f}\right\| \quad$, $\left|\tilde{y}_{f}^{(n)}\right| \leq\left\|\tilde{q}_{f}\right\|$, (70) can be rewritten as follows 


$$
\begin{aligned}
\dot{V} \leq & -\frac{1}{2} \theta_{c}\left[\lambda_{\min }(Q)+\lambda_{2}\right]\left\|\tilde{q}_{f}\right\|^{2}+\theta_{c}\left\|B_{2}^{T} Q\right\|\left\|\tilde{q}_{f}\right\|\left\|w_{1}\right\| \\
& +\left(\lambda_{3} k_{n-1}+k_{n-2}\right)\left|\delta_{f}\right|\left\|\tilde{q}_{f}\right\|+\left|\delta_{f}\right|\left\|w_{2}\right\|-\beta\left|\delta_{f}\right|^{2} \\
& +\sigma_{1}\left|\hat{T}_{1}\right|\left|\tilde{T}_{1}\right|+\sigma_{2}\left|\hat{T}_{2}\right|\left|\tilde{T}_{2}\right|+\frac{1}{\gamma_{c 1}}\left|\hat{T}_{1}\right|\left|\tilde{T}_{1}\right|+\frac{1}{\gamma_{c 2}}\left|\hat{T}_{2}\right|\left|\tilde{T}_{2}\right|
\end{aligned}
$$

From lemma 1,

$$
\begin{aligned}
\theta_{c}\left\|B_{2}^{T} Q\right\|\left\|\tilde{q}_{f}\right\|\left\|w_{1}\right\| & \leq c_{1} \theta_{c}\left\|B_{2}^{T} Q\right\|\left\|\tilde{q}_{f}\right\|\left|\tilde{T}_{1}\right|+c_{2} \theta_{c}\left\|B_{2}^{T} Q\right\||| Z \|\left|\tilde{T}_{2}\right| \\
& \leq \alpha_{4}\left\|\tilde{q}_{f}\right\|^{2}+\alpha_{1} \beta\left|\tilde{T}_{1}\right|^{2}+\alpha_{2} \beta\left|\tilde{T}_{2}\right|^{2}
\end{aligned}
$$

$$
\begin{aligned}
\left|\delta_{f}\right|\left\|w_{2}\right\| & \leq c_{1}\left|\delta_{f}\right|\left|\tilde{T}_{1}\right|+c_{2}\left|\delta_{f}\right|\left|\tilde{T}_{2}\right|+c_{3}\left|\delta_{f}\right| \\
& \leq \alpha_{4}\left|\delta_{f}\right|^{2}+c_{1}\left|\tilde{T}_{1}\right|^{2}+c_{2}\left|\tilde{T}_{2}\right|^{2}+\frac{c_{3}}{2}
\end{aligned}
$$

where $\alpha_{1}=0.5 c_{1} \theta_{c}\left\|B_{2}^{T} Q\right\|, \alpha_{2}=0.5 c_{2} \theta_{c}\left\|B_{2}^{T} Q\right\|$, $\alpha_{3}=\alpha_{1}+\alpha_{2}$ and $\alpha_{4}=c_{1}+c_{2}+0.5 c_{3}$.

Substituting (72) and (73) to (71) with the following inequalities

$$
\begin{aligned}
& \left(\lambda_{3} k_{n-1}+k_{n-2}\right) \leq\left|\delta_{f}\right||| \tilde{q}_{f}\left\|\leq \alpha_{5}\left|\delta_{f}\right|^{2}+\alpha_{5}|| \tilde{q}_{f}\right\|^{2}, \\
& \left|\hat{T}_{i}\right|\left|\tilde{T}_{i}\right| \leq \frac{1}{2}\left|\hat{T}_{i}\right|^{2}-\frac{1}{2}\left|\tilde{T}_{i}\right|^{2}(i=1,2), \\
& \frac{1}{\gamma_{c 1}}\left|\dot{T}_{1}\right|\left|\tilde{T}_{1}\right|+\frac{1}{\gamma_{c 2}}\left|\dot{T}_{2}\right|\left|\tilde{T}_{2}\right| \leq \alpha_{6}\left|\dot{T}_{1}\right|^{2}+\alpha_{6}\left|\tilde{T}_{1}\right|^{2}+\alpha_{7}\left|\dot{T}_{2}\right|^{2}+\alpha_{7}\left|\tilde{T}_{2}\right|^{2} . \\
& \text { where } \alpha_{5}=\left(\lambda_{3} k_{n-1}+k_{n-2}\right) / 2, \alpha_{6}=1 / 2 \gamma_{c 1} \text { and } \\
& \alpha_{7}=1 / 2 \gamma_{c 2} \quad \text { are positive constants, yields } \\
& \dot{V} \leq-\frac{1}{2}\left[\theta_{c} \lambda_{\min }(Q)+\theta_{c} \lambda_{2}-2 \alpha_{3}\right]|| \tilde{q}_{f} \|^{2}-\left(\frac{1}{2} \sigma_{1}-\alpha_{1}-c_{1}-\alpha_{6}\right) \\
& \quad\left|\tilde{T}_{1}\right|^{2}+\frac{c 3}{2}+\frac{1}{2} \sigma_{1}\left|T_{1}\right|^{2}+\frac{1}{2} \sigma_{2}\left|T_{2}\right|^{2}+\alpha_{6}\left|\dot{T}_{1}\right|^{2}+\alpha_{7}\left|\dot{T}_{2}\right|^{2} \\
& \quad \leq-\frac{1}{2}\left(\theta_{c} \lambda_{2}-\bar{\lambda}_{2}\right)|| \tilde{q}_{f} \|^{2}-\left(\frac{1}{2} \sigma_{1}-\bar{\sigma}_{1}\right)\left|\tilde{T}_{1}\right|^{2} \\
& \quad-\left(\frac{1}{2} \sigma_{2}-\bar{\sigma}_{2}\right)\left|\tilde{T}_{2}\right|^{2}-(\beta-\bar{\beta})\left|\delta_{f}\right|^{2}+\bar{\psi}
\end{aligned}
$$

where $\bar{\lambda}_{2}=2 \alpha_{3}+2 \alpha_{5}-\theta_{c} \lambda_{\min }(Q), \bar{\sigma}_{1}=\alpha_{1}+c_{1}+\alpha_{6}$, $\bar{\sigma}_{2}=\alpha_{2}+c_{2}+\alpha_{7}, \bar{\beta}=\alpha_{4}+\alpha_{5} \quad$ and $\bar{\psi}=\left(c_{3}+\sigma_{1}\left|T_{1}\right|^{2}+\sigma_{2}\left|T_{2}\right|^{2}\right) / 2+\alpha_{6}\left|\dot{T}_{i}\right|^{2}+\alpha_{7}\left|\dot{T}_{2}\right|^{2}$. If design parameters chosen as $\lambda_{2}>\bar{\lambda}_{2} / \theta_{c}, \sigma_{1}>2 \bar{\sigma}_{1}$, $\sigma_{1}>2 \bar{\sigma}_{2}, \beta>\bar{\beta}$. It is clear that $\dot{V}$ is negative as long as $\tilde{q}_{f}$ is outside the compact set $\Omega_{\tilde{q} f}$ defined as

$$
\Omega_{\tilde{q} f}=\left\{\tilde{q}_{f} \mid\left\|\tilde{q}_{f}\right\| \leq \sqrt{\frac{2 \bar{\psi}}{\theta_{c} \lambda_{2}-\bar{\lambda}_{2}}}\right\}
$$

According to the Lyapunov theorem [31], it can be concluded that $\hat{q}_{f}$ is bounded and converges to $\Omega_{\tilde{q} f}$. This means $\hat{q}_{f}$ is uniformly ultimately bounded (UUB). Moreover, the radius of $\Omega_{\tilde{q} f}$ can be made arbitrarily small, if $\lambda_{2}$ is chosen to be sufficiently large. Since the stable filter is in a bounded input bounded output (BIBO) form, there exist a positive constant $\rho_{\tilde{q} f}$ such as $\tilde{q}=\rho_{\tilde{q} f} \tilde{q}_{f}$ [32], thus $\tilde{q}$ is uniformly ultimately bounded (UUB).

Similarly, $\tilde{T}_{1}$ and $\tilde{T}_{2}$ are uniformly ultimately bounded (UUB) and converge to $\Omega_{\widetilde{T}_{1}}$ and $\Omega_{\widetilde{T}_{2}}$ respectively. $\Omega_{\widetilde{T}_{1}}$ and $\Omega_{\widetilde{T}_{2}}$.defined as

$$
\begin{aligned}
& \Omega_{\tilde{T}_{1}}=\left\{\tilde{T}_{1} \mid\left\|\hat{T}_{1}\right\| \leq \sqrt{\frac{2 \bar{\psi}}{\sigma_{1}-2 \bar{\sigma}_{1}}}\right\} \\
& \Omega_{\tilde{T}_{2}}=\left\{\tilde{T}_{2} \mid\left\|\tilde{T}_{2}\right\| \leq \sqrt{\frac{2 \bar{\psi}}{\sigma_{2}-2 \bar{\sigma}_{2}}}\right\}
\end{aligned}
$$

Since Assumption 1, $T_{1}$ and $T_{2}$ are bounded, $T_{1}=\hat{T}_{1}-\tilde{T}_{1}$ and $T_{2}=\hat{T}_{2}-\tilde{T}_{2}$, thus $\hat{T}_{1}$ and $\hat{T}_{2}$ are uniformly ultimately bounded (UUB).

From the inequality (74), it can be obtained

$$
\begin{aligned}
\dot{V} & \leq-\beta\left|\delta_{f}\right|^{2}+\left(\lambda_{3} k_{n-1}+k_{n-2}\right)\left|\delta_{f}\right||| \tilde{q}_{f}\left\|+\left|\delta_{f}\right|\right\| w_{2}\left\|+\theta_{c}\right\| B_{2}^{T} Q\|\| \tilde{q}_{f}\|\| w_{1} \| \\
& +\sigma_{1}\left|\hat{T}_{1}\right|\left|\tilde{T}_{1}\right|+\sigma_{2}\left|\hat{T}_{2}\right|\left|\tilde{T}_{2}\right|+\frac{1}{\gamma_{c 1}}\left|\dot{T}_{1}\right|\left|\tilde{T}_{1}\right|+\frac{1}{\gamma_{c 2}}\left|\dot{T}_{2}\right|\left|\tilde{T}_{2}\right| \\
& \leq-\beta\left|\delta_{f}\right|^{2}+\alpha_{8}\left|\delta_{f}\right|+\alpha_{9}
\end{aligned}
$$

where $\alpha_{8}, \alpha_{9} \geq 0 \quad, \quad \alpha_{8}=\left(\lambda_{3} k_{n-1}+k_{n-2}\right)\left\|\tilde{q}_{f}\right\|+\left\|w_{2}\right\|$,

$$
\begin{aligned}
\alpha_{9}= & \theta_{c}\left\|B_{2}^{T} Q\right\|\left\|\left|\tilde{q}_{f}\|\| w_{1} \|+\sigma_{1}\right| \hat{T}_{1}|| \tilde{T}_{1} \mid\right. \\
& +\sigma_{2}\left|\hat{T}_{2}\right|\left|\tilde{T}_{2}\right|+\frac{1}{\gamma_{c 1}}\left|\dot{T}_{1}\right|\left|\tilde{T}_{1}\right|+\frac{1}{\gamma_{c 2}}\left|\dot{T}_{2}\right|\left|\tilde{T}_{2}\right| .
\end{aligned}
$$

Since $\dot{T}_{1}, \dot{T}_{2}, \hat{T}_{1}, \hat{T}_{2}, \tilde{T}_{1}, \tilde{T}_{2}, \tilde{q}_{f}, w_{1}$ and $w_{2}$ are bounded,it can be known that $\alpha_{8}, \alpha_{9}$ are bounded. Based on the inequality (74), it is clear that $\dot{V}$ is negative as long as $\delta_{f}$ is outside the compact set $\Omega_{\delta_{f}}$ defined as

$$
\Omega_{\delta_{f}}=\left\{\delta_{f}|0 \leq| \delta_{f} \mid \leq \frac{-\alpha_{8}(t)+\sqrt{\alpha_{8}^{2}+4 \beta \alpha_{9}}}{2 \beta}\right\}
$$

Based on the Lyapunov theorem [31], $\delta_{f}$ is uniformly ultimately bounded and converges to compact set $\Omega_{\delta_{f}}$. According to Assumption 4, definition (33) and (34), it can be seen that $\delta_{f}(0)=0$. Based on (78), there exist 


$$
\begin{aligned}
& \Omega_{\delta_{f}(t)}= \\
& \left\{\delta_{f}(t)|0 \leq| \delta_{f}(t) \mid \leq \frac{-\alpha_{8}(t)+\sqrt{\alpha_{8}^{2}(t)+4 \beta \alpha_{9}(t)}}{2 \beta}\right\} \forall t \geq 0
\end{aligned}
$$

Since the stable filter is in a BIBO form , $\delta(0)=0$. It can be concluded that $\delta$ is uniformly ultimately bounded (UUB). Here,

$$
\begin{aligned}
& \Omega_{\delta(t)}= \\
& \left\{\delta(t)|0 \leq| \delta(t) \mid \leq \rho\left(\frac{-\alpha_{8}(t)+\sqrt{\alpha_{8}^{2}(t)+4 \beta \alpha_{9}(t)}}{2 \beta}\right)\right\} \forall t \geq 0
\end{aligned}
$$

and $\rho$ is a positive constant.

Based on (80) and lemma 2, it is clear that $\hat{s}(t)$ is uniformly ultimately bounded. The bound of $\dot{\hat{s}}(t)$ is defined as

$$
\begin{aligned}
& \Omega_{\hat{s}_{(t)}}= \\
& \left\{\hat{s}(t)|0 \leq| \hat{s}(t) \mid \leq \rho\left(\frac{-\alpha_{8}(t)+\sqrt{\alpha_{8}^{2}(t)+4 \beta \alpha_{9}(t)}}{2 \beta \lambda_{0}}\right)\right\} \forall t \geq 0
\end{aligned}
$$

According (81) the lemma 2, it is easy to know that $\xi^{(i)}(t)=[e(t)-\hat{p}(t)]^{(i)},(i=1,2, \cdots, n-1)$ is bounded, and the bound is defined as

$$
\begin{aligned}
& \Omega_{\xi^{(i)}(t)}= \\
& \left\{\xi^{(i)}(t)|0 \leq| \xi^{(i)}(t) \mid \leq \rho\left(\frac{-\alpha_{8}(t)+\sqrt{\alpha_{8}^{2}(t)+4 \beta \alpha_{9}(t)}}{2 \beta \lambda_{3} \lambda_{0}^{i}}\right)\right\}
\end{aligned}
$$

)

here $(i=1,2, \cdots, n-1), \forall t \geq 0$.

From the Assumption 4, $\xi(t)=e(t)-\hat{p}(t)$ and (82), it can be concluded that $e^{(i)}(t),(i=1,2, \cdots, n-1)$ is UUB and converges to zero in finite time $T$, where defined as

$$
\left|e^{(i)}(t)\right| \leq \rho\left(\frac{-\alpha_{8}(t)+\sqrt{\alpha_{8}^{2}(t)+4 \beta \alpha_{9}(t)}}{2 \beta \lambda_{3} \lambda_{0}^{i}}\right)
$$

$$
\text { here }(i=1,2, \cdots, n-1), \forall t \geq T \text {. }
$$

Because system (1) satisfies the Assumption 1 and 2, based on the (83), it can be seen that the system states $y, \dot{y}, \cdots, y^{(n)}$ are UUB. And it can be concluded that $\underline{x}$ is
UUB, due to the controllable $(A, B, C)$ and observable $(A, C)$. From (1) and Assumption 1, it can be seen that input $\mathrm{u}$ is UUB, furthermore, the boundedness of $\dot{u}=w$ can be obtained from (38)-(41).

Remark 2: In order to prove the stability of the proposed adaptive controller [33, 34], a three-part Lyapunov function is constructed: $V_{1}$ for observation errors with state filter, $V_{2}$ for terminal sliding mode surface contains the tracking errors, $V_{3}$ for the unknown uncertainties of the system. The key difficulty in deriving the stability theorem is scaling down the time derivative of $V$ and guaranteeing its time derivative negative.

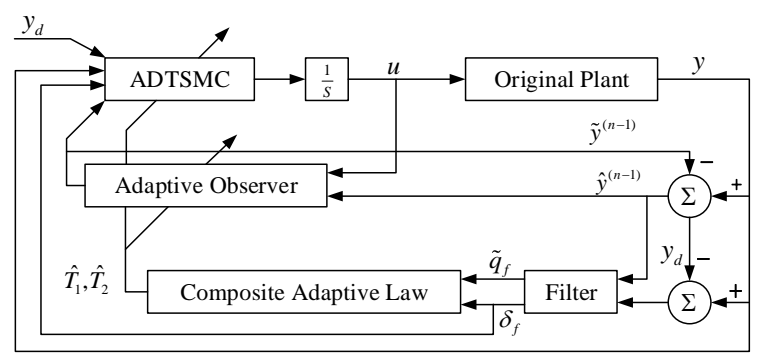

Fig. 1. Structure of Observer-based Adaptive DTSMC

\section{SIMULATION}

In this section, two numerical simulations are presented to demonstrate the properties of the proposed adaptive control algorithm. First, the proposed adaptive control algorithm is applied to a second order SISO system (see Fig. 1). The tracking performance in comparison with tracking-error-based adaptive control and observation-error-based adaptive control is presented. Next, an application to an inverted pendulum system is illustrated, and the performance of observer-based composite adaptive terminal sliding-mode control is compared with original terminal sliding-mode control.

Example 1. Consider the second order SISO system:

$$
\begin{aligned}
& \dot{x}_{1}=x_{2}, \\
& \dot{x}_{2}=f(\underline{x}, t)+g(\underline{x}, t) u(t)+d(t), \\
& y=x_{1} .
\end{aligned}
$$

where $\underline{x}=\left[\begin{array}{ll}x_{1} & x_{2}\end{array}\right]^{T}, u(t)$ is the system input, $f(\underline{x}, t)$ and $g(\underline{x}, t)$ are the dynamics which are not exactly known, $d(t)$ is the external disturbance.

In this simulation, $f(\underline{x}, t)$ and $g(\underline{x}, t)$ have the same expression as (2). The control objective is to maintain the system to track the desired trajectory $y_{d}=0$, under the condition that only $x_{1}$ and $x_{2}$ are available, the initial values is $\underline{x}(0)=\left[\begin{array}{ll}0.1 & 0\end{array}\right]^{T}$. The proposed observer and adaptive controller are designed 
as given in (23) and (37), and the design parameters used in this simulation are chosen as $k_{1}=10, k_{2}=5$, $\lambda_{2}=15, \lambda_{3}=25$.

The following cases are simulated:

Case 1. Let $f(\underline{x}, t)=30 x_{1}$ and $g(\underline{x}, t)=150$, the known parts of them are $\hat{f}(\underline{x}, t)=10$ and $\hat{g}(\underline{x}, t)=130$ respectively. No external disturbance exists in this case. Case 2. Let $f(\underline{x}, t)=[26+4 \sin (t)] x_{1} \quad$ and $g(\underline{x}, t)=140$, the known parts are $\hat{f}(\underline{x}, t)=10$ and $\hat{g}(\underline{x}, t)=130$. The external disturban $d(t)$ is defined as $d(t)=5+5 \cos (t)$.

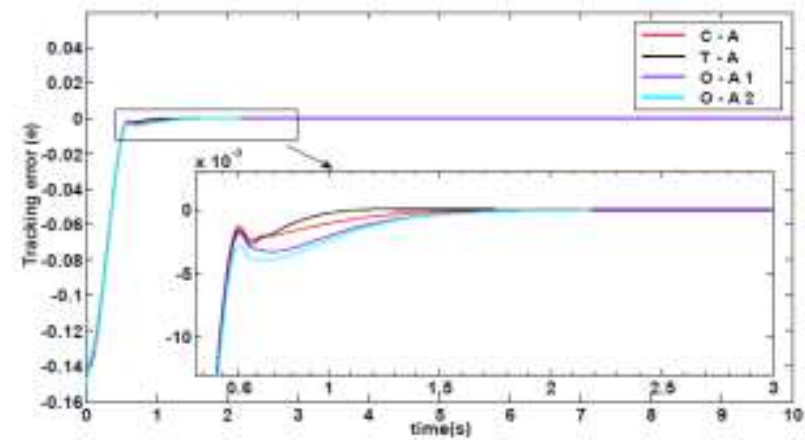

Fig. 2. The tracking-error of case 1

In the present work, C-A denote the composite adaptation proposed in this paper; $\mathrm{T}$-A denote the tracking-error based adaptation with $\gamma=800$; O-A1 denote the observation based adaptation with $\gamma=800$, $\theta=500$; O-A1 denote the observation based adaptation with $\gamma=800, \theta=250$.

Figs. 2. and 3. compare the tracking error of the tracking error-based adaptive controller with adaption gain with the observer error-based adaptive controller with two different compensation gains $\theta=250$ and $\theta=500$. It can be seen that the tracking error converged to a small bound in time $T=0.6 \mathrm{~s}$.

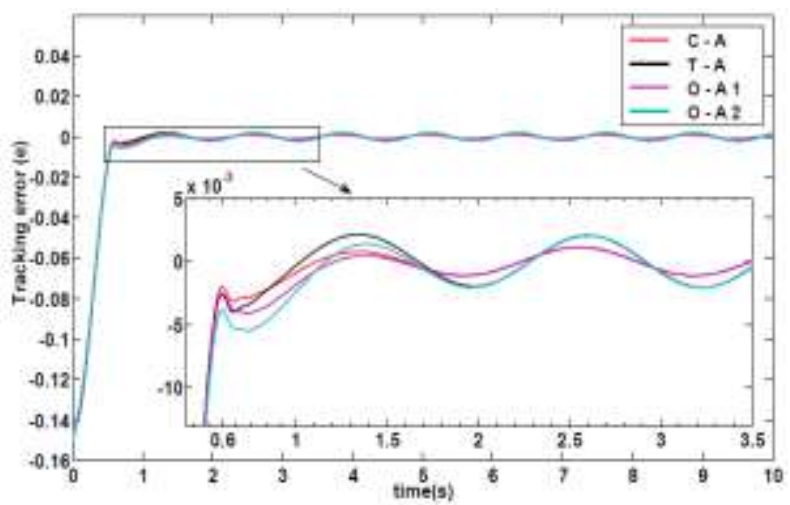

Fig. 3. The tracking-error of case 2

According to (77) - (83), it can be seen that the bound $\left|e^{(i)}(t)\right|$ relates to the adaption error. That means the good tracking performance can be obtained by a stable and fast adaption. From Fig. 2, when the uncertainties are time-invariable, the tracking performance of our proposed adaptive law is better than it of the observation error-based adaptive law, since the composite adaptive law extracts parameter information from both the tracking-error and the observation-error. It is not as good as the tracking error-based adaptive law, due to the signal weakening which is caused by the composite adaptive law. However, for this property, the proposed adaptive law has better robustness to fast time-varying uncertainties $d(t)$ than tracking error-based adaptive law (see Fig. 3).

Example 2. Consider the inverted pendulum system. Let $x_{1}=\theta$ be the angle of the pendulum with respect to the vertical line and $x_{2}=\dot{\theta}$. The dynamic equations of the inverted pendulum system are expressed as follows

$$
\begin{aligned}
& \dot{x}_{1}=x_{2}, \\
& \dot{x}_{2}=\frac{(M+m) g \sin x_{1}-m l x_{2}^{2} \cos x_{1} \sin x_{1}+u \cos x_{1}}{4 l(M+m) / 3-m l \cos ^{2} x_{1}}+d(t), \\
& y=x_{1} .
\end{aligned}
$$

where $\mathrm{g}$ is the acceleration due to gravity, $M$ is the mass of the cart, $m$ is the mass of the pole, $l$ is the half length of the rod, $y$ is the system output, $u$ is the applied force ( the control signal ), and $d(t)$ is the external disturbance.

In this simulation, it is assumed that the external disturbance $d(t)$ is a band-limited white noise with sampling period 0.001 whose magnitude is \pm 2 . Another system parameters are given as $M=1+0.5 \cos (3 t), m=0.1+0.25 \sin (5 t), l=0.5$. The control objective is to keep the system tracking the desired trajectory $y_{d}=0.1 \sin (t)$ under the condition that only the states $\mathrm{x} 1$ and $\mathrm{x} 2$ are measurable and the initial values $\underline{x}(0)=\left[\begin{array}{ll}0.15 & 0\end{array}\right]^{T}$.

The observer-based adaptive DTSMC is designed as given in (23), (37) - (41), (52) and (53) , and the design parameters used in this simulation are chosen as $k_{1}=5$, $k_{2}=1, \lambda_{2}=10, \lambda_{3}=15$. In order to illustrate the effectiveness of the proposed method, we randomly choose the initial values of $M=1, m=1$ and $l=1$ (i.e. there is no previous knowledge of the system nonlinearities).

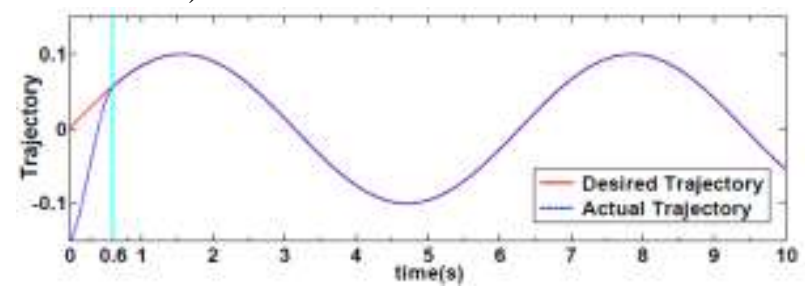


Fig. 4. The tracking performance of proposed OADTSMC

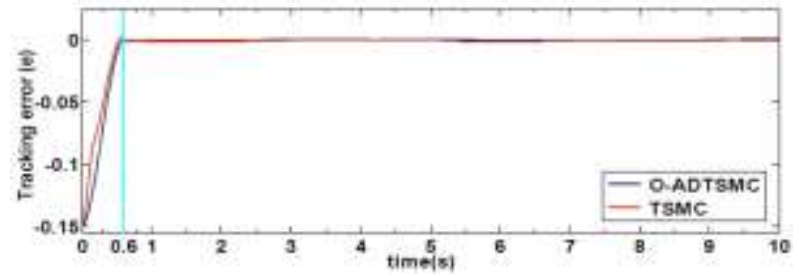

Fig. 5. Comparison of the tracking errors between $\mathrm{O}-$ ADTSMC and TSMC

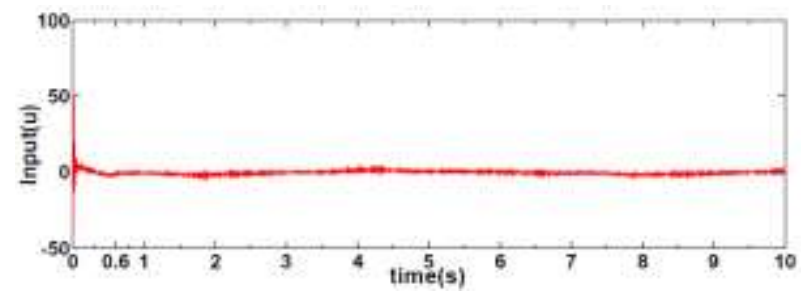

Fig. 6. The control input of Observer-based Adaptive DTSMC

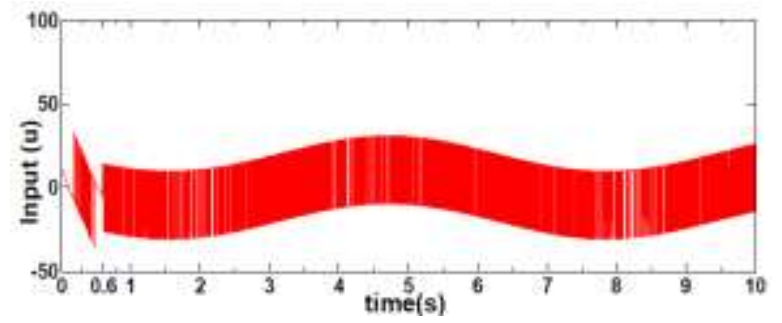

Fig. 7. The control input of TSMC

To compare the control performance, this system is also controlled by the original terminal sling mode controller with control gain $K_{T S M C}=10$,boundary layer width $\phi=0.03$ and $T=0.6$.

As shown in Fig. 5, the performance of Observerbased Adaptive DTSMC is similar to it of TSMC. However, from Figs. 6 and 7, it is clear that the chattering of control input generated by Observer-based Adaptive DTSMC is reduced effectively. Therefore, the observerbased dynamic sliding-mode control which proposed in this paper has a good robustness to uncertainties, immunity to the noise and effective reduction of input chattering.

\section{CONCLUSION}

In this paper, the proposed observer-based composite adaptive terminal sliding-mode control has good practicability. Based on the algebraic Lyapunov equation, an adaptive observer which dispense with the famous SPR condition is designed. By employing an appropriate state filter and a new function $p(t)$,both the filtering observation error vector and a new terminal sliding-mode surface which is available for the state-unavailable systems can be obtained. The stability analysis and numerical simulations are provided to illustrate stability, fast adaptation, good robustness and effectively reduction of input chattering by using the proposed controller.

\section{ACKNOWLEDGMENT}

The authors would like to express their gratitude for the reviewers's constructive comments.

\section{CONFLICTS OF INTEREST}

The authors declare that there are no conflicts of interest regarding the publication of this paper.

\section{REFERENCES}

[1] Y. Wei, J.H. Park and J. Qiu, "Sliding mode control for semi-Markovian jump systems via output feedback," Automatica, vol.81, pp.133-141, April 2017.

[2] W. Qi and G. Zong, "Observer-based adaptive SMC for nonlinear uncertain singular semi-Markov jump systems with applications to DC motor," IET Control Theory and Applications, vol.11, pp.15041513, 2017.

[3] K. Park and T. Tsuji, "Terminal sliding mode control of second-order nonlinear uncertain systems," Int. J. Robust Nonlinear Control, vol. 9, pp. 769-780, 1999.

[4] Y. Feng, X. Yu, and Z. Man, "Nonsingular terminal sliding mode control of rigid robot manipulators," Automatica, vol. 38, pp. 2159-2167, 2002.

[5] J. Liu and F. Sun, "A novel dynamic terminal sliding mode control of uncertain nonlinear systems," Journal of Control Theory and Applications, vol. 5, no. 2, pp. 189-193, 2007.

[6] M. Chen, Q. Wu, and R. Cui, "Terminal sliding mode tracking control for a class of SISO uncertain nonlinear systems," ISA Transactions, vol. 52, pp. 198-206, 2013.

[7] J. Xiong and G. Zhang, "Global fast dynamic terminal sliding mode control for a quadrotor UAV," ISA Transactions, vol. 66, pp. 233-240, 2017.

[8] M. Zak, "Terminal attractors for addressable memory in neural networks," Physics Letter, vol.133, no.12, pp.18-22, 1988.

[9] C. Yu, M. Xie, and J. Xie, "Sliding mode tracking control of nonlinear system with non-matched uncertainties," Journal of Shanghai Jiaotong University, vol. 35, no. 8, pp. 1141-1143, 2001.

[10] S. Mobayen and F. Tchier, "A novel robust adaptive second-order sliding mode tracking control technique for uncertain dynamical systems with matched and unmatched disturbances," International Journal of Control, Automation and Systems, vol. 15, no. 3, pp. 1197-1106, 2017.

[11] X. Ma, F. Sun, Hong, B. Li, and B. He, "Neuralnetwork-based integral sliding-mode tracking 
control of second-order multi-agent systems with unmatched disturbances and completely unknown dynamics," International Journal of Control, Automation and Systems, vol. 15, no. 3, pp. 19251935, 2017.

[12] M. Chen, C. Chen, and F. Yang, “An LTR-observer based dynamic sliding mode control for chattering reduction," Automatica, vol. 453, pp. 1111-1116, 2007.

[13] J. Slotine and W. Li, Applied Nonlinear Control, Prentice-Hall, Englewood Cliffs, NJ, 1991.

[14] J. Nakanishi, J. Farrell, and S. Schaal, "Composite adaptive control with locally weighted statistical learning," Neural Networks, vol. 18, pp. 71-90, 2005.

[15] P. Ioannou and J. Sun, Robust Adaptive Control, Prentice Hall, Englewood Cliffs, NJ, 1996.

[16] Y. Leu, T. Lee, and W. Wang, "Observer-based adaptive fuzzy-neural control for unknown nonlinear dynamical systems," IEEE Trans. Systems Man Cybernet. Part B: Cybernet, vol. 29, pp. 583$591,1999$.

[17] C. Kung and T. Chen, "Observer-based indirect adaptive fuzzy sliding mode control with state variable filter for unknown nonlinear dynamical systems," Fuzzy Set and Systems, vol. 155, pp. 292308, 2005.

[18] A. Boulkroune, M. Tadjine, M. M'Saad, and M. Farza, "How to design a fuzzy adaptive controller based on observers for uncertain affine nonlinear systems," Fuzzy Set and Systems, vol. 159, pp. 926948, 2008.

[19] C. Hyun, C. Park, and S. Kim, "Takagi-Sugeno fuzzy model based indirect adaptive fuzzy observer and controller design," Information Sciences, vol. 180, pp. 2314-2327, 2010.

[20] Y. Wang, T. Chai, and Y. Zhang, "State observerbased adaptive fuzzy output-feedback control for a class of uncertain nonlinear systems," Information Sciences, vol. 180, pp. 5029-5040, 2010.

[21] Y. Wei, J. H. Park, H. R. Karimi, and Y. C. Tian, "Improved stability and stabilization results for stochastic synchronization of continuous-time semiMarkovian jump," IEEE Transactions on Neural Networks and Learning Systems, vol.29, no.6, pp.2488-2501, 2018.

[22] Y. Wei, J. Qiu, and H. R. Karimi, "Fuzzy-affinemodel-based memory filter design of nonlinear systems with time-varying delay," IEEE Transactions on fuzzy systems, vol.26, no.2, pp.504$517,2018$.

[23] M. Zeinali and L. Notash, "Adaptive sliding mode control with uncertainty estimator for robot manipulators," Mechanism and Machine Theory, vol. 45, pp. 80-90, 2010.

[24] C. Veluvolu and D. Lee, "Sliding mode high-gain observers for a class of uncertain nonlinear systems," Applied Mathematics Letters, vol. 24, pp. 329334,2011.

[25] H. Li, P. Shi, D. Yao, and L. Wu, "Observer-based adaptive sliding mode control for nonlinear
Markovian jump systems," Automatica, vol. 64, pp. 133-142, 2016.

[26] C. C. Hang, "On state variable filters for adaptive system design," IEEE Trans. Automatic Control, vol. 21, no. 6, pp. 874-876, 1976.

[27] E. Lavretsky, "Combined/composite model reference adaptive control," IEEE Trans. Automatic Control, vol. 54, no. 11, pp. 2692-2697, 2009.

[28] Y. Pan, Y. Zhou, T. Sun, and J. Meng, "Composite adaptive fuzzy $H \propto$ tracking control of uncertain nonlinear systems," Neurocomputing, vol. 99, pp. 15-24, 2013.

[29] P. Ioannou and P. Kokotovic, "Instability analysis and improvement of robustness of adaptive control," Automatica, vol. 20, pp. 583-594,1984.

[30] S. Blazic, D. Matko, and I. Skrjanc, "Adaptive law with a new leakage term," IET Control Theory Appl., vol. 4, no. 9, pp. 1533-1542, 2010.

[31] H. Khalil, Nonlinear Systems, Prentice-Hall, Englewood Cliffs, NJ, 2002.

[32] H. Yong, L. Lewis, and T. Chaouki, "A dynamic recurrent neural-network-based adaptive observer for a class of nonlinear systems," Automatica, vol. 33, pp. 1539-1543, 2003.

[33] B. B. Letswamotse, R. Malekian, C.Y. Chen, K.M. Modieginyane, "Software Defined Wireless Sensor Networks (SDWSN): A Review on Efficient Resources, applications and technologies, Journal of Internet Technology.

[34] X. Jin, J. Shao, X. Zhang, W. An, R. Malekian, "Modeling of nonlinear system based on deep learning framework", Nonlinear Dynamics, Springer, Vol.84, No. 3, pp.1327-1340, 2016. 